\title{
Diferencia salarial entre hombres y mujeres: Un estudio de Guatemala desde la perspectiva de las ocupaciones en el 2019
}

DOI del artículo: 10.36631/ECO.2021.24.02 Artículo de investigación científica y tecnológica

Dayana Alejandra Gamboa Palacios
Licenciada en Economía Empresarial por la Universidad Rafael Landívar
Correo electrónico: dagamboagt@gmail.com
Fecha de recepción: 10/1/2021
Fecha de aceptación: 25/2/2021

\section{Resumen}

Esta investigación tiene como propósito analizar las diferencias salariales desde una perspectiva de las ocupaciones en el 2019. A partir de dicho enfoque, se contribuye a comprender de una mejor manera el fenómeno de desigualdades entre los ingresos de mujeres y hombres. El enfoque metodológico se basa en la descomposición de Oaxaca-Blinder. Los resultados revelan la presencia de una diferencia salarial causada por discriminación por sexo en el mercado laboral guatemalteco, lo que afecta a las mujeres, a pesar de contar con mejores características personales.

Palabras clave: diferencia salarial, mercado laboral, ocupaciones, Guatemala 


\title{
WAGE GAPS BETWEEN MEN AND WOMEN: A STUDY OF GUATEMALA IN 2019 FROM AN OCCUPATIONAL PERSPECTIVE
}

\author{
Scientific and technological research paper
}

\section{Dayana Alejandra Gamboa Palacios}

Business Economics degree, Universidad Rafael Landívar

Email: dagamboagt@gmail.com

Reception date: $1 / 10 / 2021$

Acceptance date: 2/25/2021

\section{Abstract}

The purpose of this investigation is to analyze salary differences during 2019 from a job or occupational perspective. Said perspective provides a better understanding of the phenomenon of income inequality between men and women. The methodological approach is based on the Oaxaca-Blinder decomposition method. The results reveal a wage gap in the Guatemalan labor market caused by sex discrimination, which affects mostly females even though they posses better personal attributes.

Keywords: salary or wage gaps, labor market, jobs, Guatemala 


\section{Introducción}

El presente estudio tuvo como objetivo estimar las brechas salariales por sexo según las distintas ocupaciones y explicar la composición de dichas brechas. La profundización en los determinantes del salario es de suma importancia, dado que permite que instituciones públicas y privadas encuentren mecanismos de asignación más eficiente y equitativa de los recursos en una sociedad. La base teórica del estudio es la acumulación de capital, ya que permite analizar los determinantes de los ingresos de los individuos, la desigualdad salarial y la manera en que la masa salarial es distribuida. Es de suma importancia mencionar que, en dicho modelo, la educación y la experiencia son los componentes más importantes.

Para lo anterior, se utilizó el método de descomposición de Oaxaca-Blinder, el cual se divide en un componente explicado por las características individuales de las personas, y en otro componente no explicado, al cual se le atribuye la discriminación en el mercado laboral. El aporte que se busca con la presente investigación es contribuir al debate de los diferenciales salariales, al proporcionar evidencia empírica que permita comprender de mejor manera el fenómeno e, idealmente, brindar más elementos para el desarrollo de una política pública orientada a alcanzar una mayor igualdad entre los ingresos de mujeres y hombres. 


\section{Método}

Para la presente investigación, se definieron como sujetos de análisis todos aquellos individuos de la Encuesta Nacional de Empleo e Ingresos (ENEI) 1-2019 que componen la población en edad de trabajar (quince años en adelante), ocupados, económicamente activos y que percibieron un salario (Instituto Nacional de Estadística [INE], 2019). A partir de dicha variable, se construyó el agregado de los ingresos como la suma del producto de los doce sueldos anuales más los ingresos por horas extras, comisiones, ingreso por trabajar en su periodo vacacional, bono 14, aguinaldo, bono vacacional, décimo quinto sueldo, subsidio de alimentación, vivienda, combustible, parqueo y bono de productividad.

La variable dependiente es el agregado de ingresos anteriormente descrito, el cual es una variable logarítmica que se encarga de medir el ingreso anual total de los individuos. Por otro lado, las variables independientes se refieren al nivel de escolaridad del individuo, la edad y edad al cuadrado; asimismo, se tienen tres variables dicotómicas que representan el sexo del individuo, si pertenece al grupo étnico indígena o no indígena y si en su empleo cuenta o no con contrato.

Para el análisis, se utilizó el método de descomposición de Oaxaca-Blinder, desarrollado por los economistas Alan Blinder y Ronald Oaxaca, en 1970. Se eligió dicha metodología ya que permite encontrar las razones y fuentes de la brecha salarial por sexo.

Esta metodología permite obtener la descomposición de la brecha salarial. La descomposición consiste en dividir la diferencia salarial en dos partes. La primera parte es la «explicable», pues demuestra el peso que tienen las características personales de los individuos en las diferencias salariales: entre estas cualidades, está la edad, experiencia laboral, nivel de estudios, etcétera. Por otro lado, el segundo componente es la parte «no explicable», a la cual comúnmente se le atribuye la discriminación presente (Ortiz, 2017).

Como se mencionó anteriormente, con el primer paso, se busca estimar una regresión para hombres y mujeres.

Ecuación 1: $\quad \ln \mathrm{Sal}_{\mathrm{m}}=\mathrm{b}_{\mathrm{m}} \mathrm{X}_{\mathrm{m}}+\mathrm{e}_{\mathrm{i}}$

Ecuación 2: $\quad \operatorname{lnSal}_{\mathrm{f}}=\mathrm{b}_{\mathrm{f}} \mathrm{X}_{\mathrm{f}}+\mathrm{e}_{\mathrm{i}}$

El subíndice «m» corresponde a todas las variables para la muestra de los hombres, por otro lado, el subíndice «f» representa a toda la muestra femenina. En la primera ecuación, $\mathrm{InSal}_{\mathrm{m}}$ se refiere al logaritmo natural del salario de los hombres; 
$\mathrm{b}_{\mathrm{m}}$ representa todos los coeficientes y es así un vector del sexo masculino; $\mathrm{X}_{\mathrm{m}}$ representa todas las variables explicativas correspondientes a los hombres $\mathrm{y}$ $\mathrm{e}_{\mathrm{i}}$ es el término de error.

Por otro lado, en la Ecuación 2, InSal se refiere al logaritmo natural del salario de las mujeres; $b_{f}$ representa todos los coeficientes y es así un vector correspondiente al sexo femenino; $X_{f}$ representa todas las variables explicativas de la mujer $y$ e $e_{i}$ es el término de error. Las ecuaciones 1 y 2 se realizan para cada una de las ocupaciones $y$, en total, son veinte regresiones.

La ecuación de la descomposición de Oaxaca-Blinder incluye los valores correspondientes a los interceptos y coeficientes de las regresiones, así como los valores promedio de las variables independientes. Se muestra a continuación:

$$
\mathrm{DO}=\operatorname{lnSal_{m}}-\operatorname{lnSal_{f}}=\mathrm{b}_{\mathrm{m}}\left(\mathrm{X}_{\mathrm{m}}-\mathrm{X}_{\mathrm{f}}\right)+\left[\left(\alpha_{\mathrm{m}}-\alpha_{\mathrm{f}}\right)+\mathrm{X}_{\mathrm{f}}\left(\mathrm{b}_{\mathrm{m}}-\mathrm{b}_{\mathrm{f}}\right)\right]
$$

La ecuación DO, por descomposición Oaxaca-Blinder, establece la diferencia logarítmica natural entre el ingreso salarial anual promedio de los hombres y el de las mujeres. Según se mencionó, la descomposición de Oaxaca-Blinder demuestra la diferencia salarial por medio de dos partes: la primera es explicada por características personales, lo cual, en la ecuación DO, está descrito como $b_{m}\left(X_{m}-X_{f}\right)$; y la segunda parte, como $\left[\left(\alpha_{m}-\alpha_{f}\right)+X_{f}\left(b_{m}-b_{f}\right)\right]$, que representa la proporción de la diferencia salarial explicable por la discriminación existente.

\section{Datos}

El marco maestro de muestreo de la ENEI 1-2019 fue de 15511 sectores y 2127915 viviendas, con una muestra maestra de 5000 sectores y 772490 viviendas. La muestra de la ENEI 1-2019 fue de 365 sectores, 5227 viviendas y 22097 personas, lo que fue un total de 10679 hombres y 11418 mujeres (INE, 2019). Dicho tamaño de muestra es de utilidad, dado que cuenta con un factor de expansión que es útil para hacer la representación a nivel nacional.

\section{Resultados}

A partir de las estimaciones realizadas con la base de datos de la ENEI 1-2019, se puede decir que las mujeres en Guatemala ganan, en promedio, menos que los hombres. Las razones salariales mujeres-hombres, en nueve ocupaciones, se encuentran en un rango intercuartílico de $56.67 \%$ a $89.34 \%$, con una media de $79.20 \%$. Es importante mencionar que para la ocupación de militares no se pudo realizar ninguna estimación de diferencias salariales, debido a la ausencia de información sobre mujeres que pertenecen a dicha rama; por lo tanto, en esta investigación, no podrá revelarse cómo se comporta el mercado laboral de la ocupación militar en Guatemala. Cabe mencionar que, dentro del estudio, 
se especifica qué proporción de la diferencia de ingresos que se presenta en los mercados laborales se debe en parte a las diferencias en acumulación de capital humano y a la discriminación.

\section{Figura 1. Participación laboral femenina (PLF) en cada ocupación}

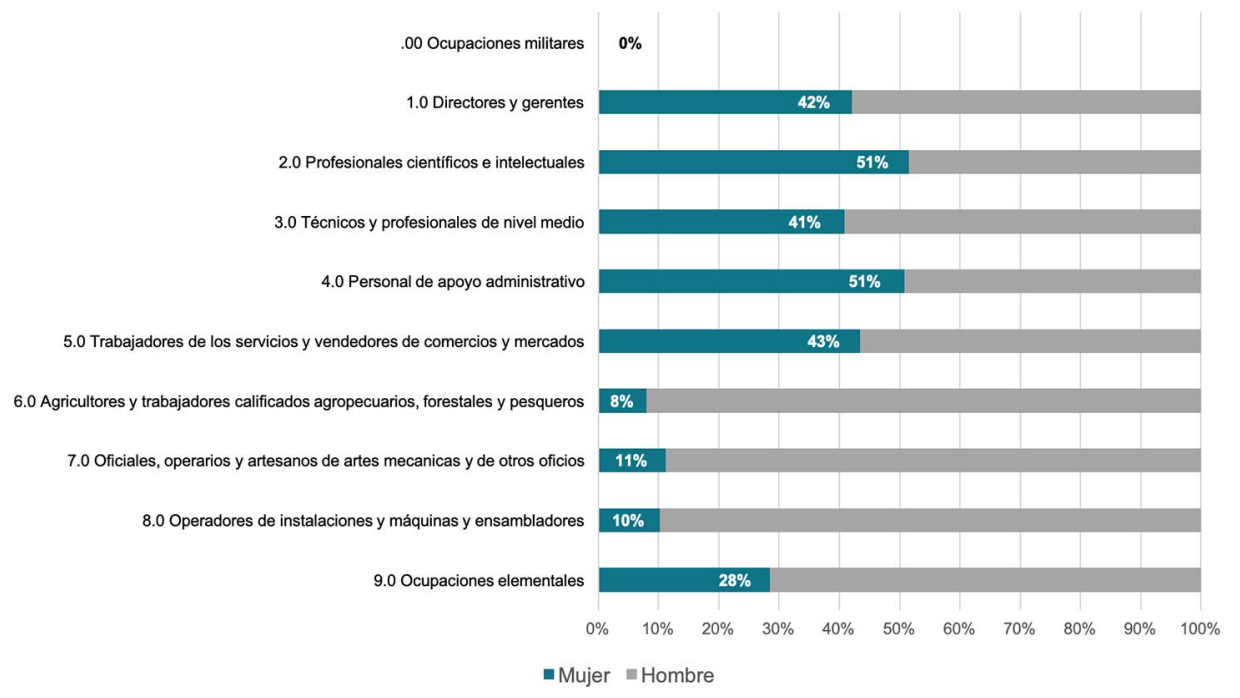

Figura 1. La gráfica representa el porcentaje de mujeres que laboran en cada uno de los nueve sectores que formaron parte de la presente investigación. Fuente: elaboración propia, con base en datos de ENEI 1-2019.

En la figura 1, se puede observar la concentración de la PLF por cada ocupación: al tomar en cuenta la codificación asignada por la Clasificación Internacional Uniforme de las Ocupaciones (CIUO-08) de la Organización Internacional del Trabajo (2016), en las ocupaciones 2 y 4 es donde se concentran los mayores porcentajes de la fuerza femenina, caso contrario en las ocupaciones 6, 7 y 8, con menores porcentajes $y$, a un nivel extremo, 0 , donde la fuerza laboral femenina es inexistente.

En la figura 2 se presenta la proporción de los ingresos salariales promedio de mujeres y hombres por ocupación, el eje horizontal sigue la codificación asignada por la CIUO-08. La gráfica permite analizar de mejor manera la diferencia salarial existente en cada ocupación en el mercado laboral guatemalteco, en el que las mujeres perciben un salario promedio menor que el de los hombres. 
Figura 2. Ingresos salariales anuales, promedio por sexo y ocupación (montos expresados en quetzales)

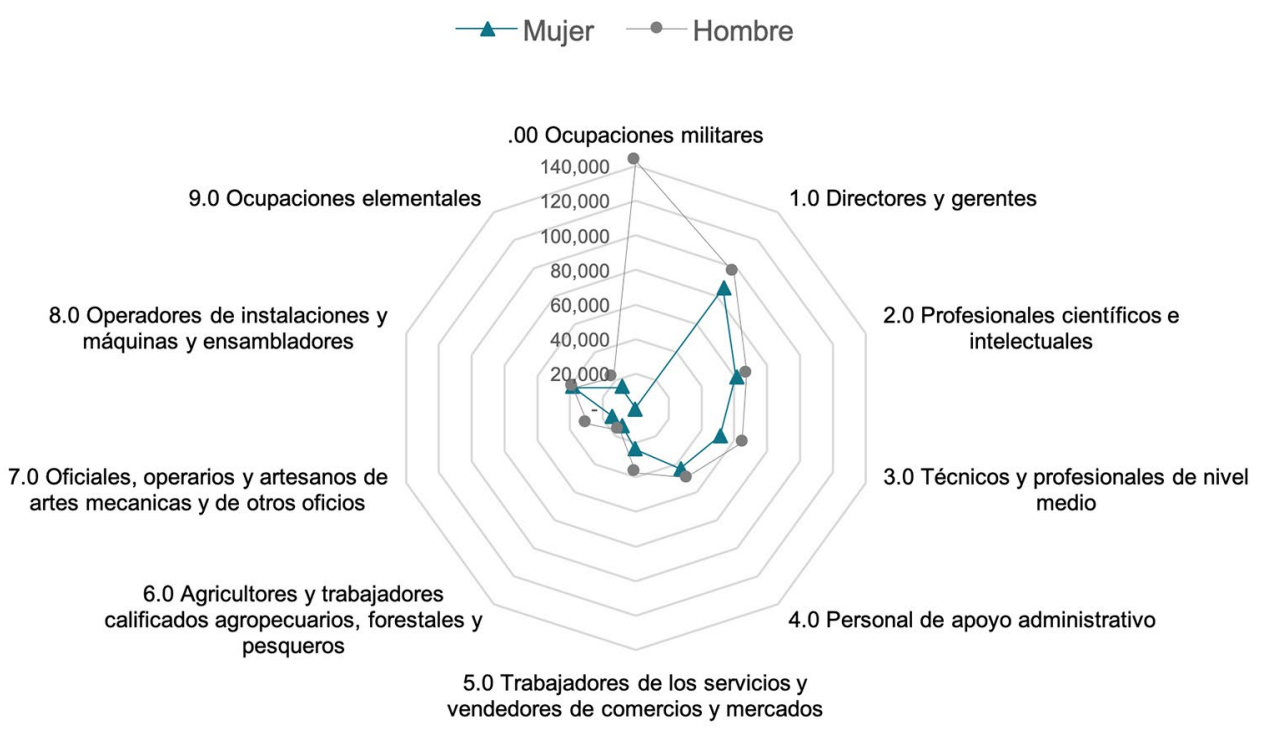

Figura 2. La gráfica muestra la diferencia salarial existente entre hombres y mujeres que ocupan una determinada posición en el mercado laboral guatemalteco, denotándose que el género masculino percibe mayores ingresos por ejercer la misma ocupación. Fuente: elaboración propia, con base en datos de ENEl 1-2019.

Para analizar los salarios y su relación con el nivel de vida de las y los trabajadores, se estableció una estratificación basada en el costo de la Canasta Básica Alimentaria (CBA) y Canasta Ampliada, publicada en marzo de 2019 por el Instituto Nacional

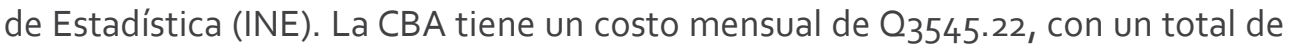
Q42 542.64 anuales, mientras el costo de la CA es de Q8185.68 al mes y Q97 544.16 por año. Se clasifican como salarios bajos aquellos que son inferiores o iguales al costo anual de la CBA; los salarios medios se encuentran por encima del costo anual de la CBA y son inferiores o iguales al costo anual de la CA, es decir, entre Q42 542.65 y Q97 544.16; por último, los salarios altos son aquellos superiores al costo anual de la CA.

A partir de la clasificación anteriormente descrita, las ocupaciones 5 a 9, en promedio, se tipifican como salarios bajos, de la 1 a 4 como medios, y únicamente la ocupación 0 como alto.

Al observar de manera combinada la información de la PLFy los ingresos promedio, por ocupación, se puede observar que la PLF se concentra en las ocupaciones con salarios bajos y medios, con $66 \%$ y $34 \%$, respectivamente. Es importante mencionar que las mujeres con más años de estudios formales se ubican en las ocupaciones con salarios medios. 
Figura 3. Razón por ocupación

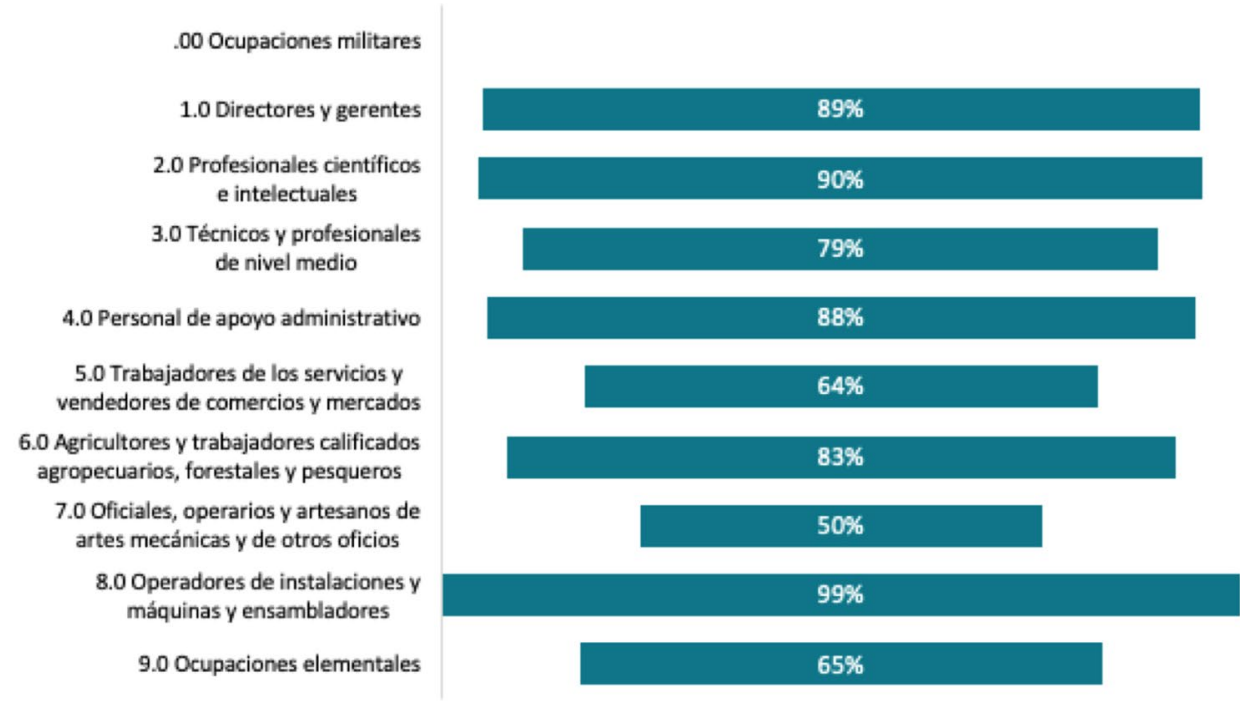

Figura 3. La figura muestra la brecha porcentual existente entre el salario que percibe el género femenino en comparación con el que percibe el género masculino al ocupar la misma posición. Fuente: elaboración propia, con base en datos de ENEl 1-2019.

La figura 3 muestra la diferencia salarial entre cada ocupación: la ocupación de oficiales, operarios/as y artesanos/as de artes mecánicas y de otros oficios es donde se encuentra la brecha más grande, de $50 \%$, lo que demuestra que, por cada $\mathrm{Q}_{100}$ que un hombre percibe, la mujer, en promedio, percibe $\mathrm{Q}_{50}$. Mientras, la ocupación con menor brecha salarial es la de operadores/as de instalaciones y máquinas y ensambladores/as, donde, por cada Q100 que un hombre recibe como salario, la mujer percibe Qو9. Sin embargo, dicha brecha salarial no permite conocer si la diferencia salarial es causada o no por la presencia de discriminación, por lo que se utiliza la metodología de Oaxaca-Blinder para realizar un estudio más preciso sobre la desigualdad y la descomposición.

Los resultados obtenidos de la descomposición de Oaxaca-Blinder permiten observar la ponderación de la diferencia salarial que puede ser explicada por las diferencias en las características personales. Para ello, se utilizaron las variables de edad, edad al cuadrado, cantidad de años de educación formal, si la persona se autodenomina indígena o no indígena, si reside en el área urbana o rural y si cuenta o no con contrato. Por otro lado, a la parte no explicada se le atribuye el resultado de prácticas discriminatorias que puedan presentarse en la ocupación. A continuación, se presenta la información obtenida de cada variable de manera desglosada. 
A partir de las regresiones realizadas en todas las ocupaciones, se demuestra que existe una relación positiva con los ingresos salariales y el total de años de educación formal, el cual juega un papel de suma importancia. La variable de la edad, utilizada como proxy de la experiencia laboral, tiene un efecto positivo en los salarios, sin embargo, presenta una tasa marginalmente decreciente, tal y como se observa en la variable de edad al cuadrado. No obstante, dicho axioma no es válido para la regresión de directores masculinos, oficiales y técnicos femeninos, donde la variable independiente de edad es negativa y edad al cuadrado, positiva. Por otro lado, se presenta una relación positiva, en promedio, entre los ingresos percibidos y residir en el área urbana; se exceptúan de dicha afirmación aquellas regresiones de las mujeres de ocupaciones agrícolas y directoras, mientras que, en la ocupación de operadores/as, comprende a ambos sexos.

En la variable de la pertenencia étnica no indígena existe una relación positiva, en promedio, con los ingresos salariales; las regresiones que se exceptúan de dicha relación son en los hombres que se encuentran en las ocupaciones de directores y técnicos. Es importante mencionar que los ingresos de las personas que cuentan con un contrato tienden a ser mayores que los de quienes no poseen uno.

Con los resultados obtenidos de las medias ponderadas y las regresiones calculadas de cada regresión, se procedió a calcular las respectivas descomposiciones de Oaxaca-Blinder; la siguiente tabla contiene la información resumida de las mismas.

Tabla 1

Resumen de los resultados de Oaxaca-Blinder de las diferencias salariales

\begin{tabular}{|c|c|c|c|}
\hline Criterios & $\begin{array}{l}\text { Directores/as y } \\
\text { gerentes }\end{array}$ & $\begin{array}{l}\text { Profesionales } \\
\text { científicos/as e } \\
\text { intelectuales }\end{array}$ & $\begin{array}{l}\text { Técnicos y profesionales } \\
\text { de nivel medio }\end{array}$ \\
\hline Diferencia & 0.0702275 & 0.1158768 & 0.1684524 \\
\hline Explicada & $-108 \%$ & $-24 \%$ & $-18 \%$ \\
\hline No explicada & $208 \%$ & $124 \%$ & $118 \%$ \\
\hline Criterios & $\begin{array}{l}\text { Personal } \\
\text { de apoyo } \\
\text { administrativo }\end{array}$ & $\begin{array}{l}\text { Trabajadores/as } \\
\text { de los servicios } \\
\text { y vendedores/as } \\
\text { de comercios y } \\
\text { mercados }\end{array}$ & $\begin{array}{l}\text { Agricultores/as } \\
\text { y trabajadores/ } \\
\text { as calificados/as } \\
\text { agropecuarios/as, } \\
\text { forestales y pesqueros/as }\end{array}$ \\
\hline Diferencia & 0.1334581 & 0.567491 & 0.1317565 \\
\hline Explicada & $4 \%$ & $38 \%$ & $5 \%$ \\
\hline No explicada & $96 \%$ & $62 \%$ & $95 \%$ \\
\hline
\end{tabular}




\begin{tabular}{|c|c|c|c|}
\hline Criterios & $\begin{array}{l}\text { Oficiales, } \\
\text { operarios/as } \\
\text { y artesanos/ } \\
\text { as de artes } \\
\text { mecánicas y de } \\
\text { otros oficios }\end{array}$ & $\begin{array}{l}\text { Operadores/as } \\
\text { de instalaciones } \\
\text { y máquinas y } \\
\text { ensambladores/as }\end{array}$ & $\begin{array}{l}\text { Ocupaciones } \\
\text { elementales }\end{array}$ \\
\hline Diferencia & 0.9640175 & -0.0793548 & 0.5332735 \\
\hline Explicada & $25 \%$ & $231 \%$ & $4 \%$ \\
\hline No explicada & $75 \%$ & $-131 \%$ & $96 \%$ \\
\hline
\end{tabular}

Fuente: elaboración propia, con base en datos de ENEl 1-2019.

A partir de la información obtenida por medio de las regresiones realizadas y la descomposición de Oaxaca-Blinder, se observa que las brechas salariales con base logarítmica en las ocupaciones se encuentran en un rango entre $0.093 \mathrm{y}$ 0.550 , con una media de 0.28 . Las ocupaciones que se encuentran dentro del rango mencionado son agrícolas, ocupaciones elementales, personal de apoyo, científicos y técnicos.

La ocupación agrícola presenta un coeficiente de diferencia salarial logarítmica de 0.132 . Según la parte explicada, las mujeres deberían de ganar 0.007 (más del $5 \%$ ) con sus atributos productivos, sin embargo, reciben 0.125 (menos del 95\%), correspondiente a la parte no explicada atribuible a la discriminación. Se observa que las variables que tienen mayor relación positiva en la parte explicada es la etnicidad no indígena y la edad; sin embargo, esta última presenta rendimientos marginales decrecientes, lo cual es observable en la variable de edad al cuadrado. En la ocupación agrícola, tienden a emplear más hombres no indígenas que mujeres no indígenas, mujeres del área urbana que hombres del área urbana, así como tienden a contar con un contrato laboral.

Las ocupaciones elementales tienen una brecha salarial logarítmica de 0.533 : la sumatoria de la diferencia explicada es 0.020 , lo que representa que las mujeres deberían de ganar un $4 \%$ más, relacionado con sus atributos productivos, sin embargo, perciben 0.513 puntos logarítmicos, es decir, $96 \%$ menos, lo que puede ser atribuible a la discriminación. Las variables de la parte explicada con mayor ponderación son contar con contrato y educación; en promedio, son los hombres con mayores niveles de educación y mayor proporción quienes cuentan con contrato.

Por otro lado, el personal de apoyo administrativo tiene una diferencia salarial logarítmica de 0.133 , donde las mujeres deberían de percibir un $4 \%$ más en sus salarios, relacionado con sus características personales, según el coeficiente de 0.005 de la parte explicada; en cambio, reciben un $96 \%$ menos, por el coeficiente de la parte no explicada de $\mathbf{0 . 1 2 9}$, lo que puede ser a causa de las prácticas 
discriminatorias. En dicha ocupación, las variables con relación positiva con la parte explicada son contar con un contrato, la edad y autodenominarse como no indígena. De las y los empleados de dicha ocupación, las mujeres tienen, en promedio, más años de escolaridad que los hombres; además, una mayor proporción de mujeres que hombres son del área urbana, tienen contrato y son no indígenas.

La ocupación de profesionales científicos/as e intelectuales tiene una diferencia salarial de 0.116 puntos logarítmicos, compuesta por una parte explicada que demuestra que las mujeres deberían de ganar -0.028 (más $24 \%$ ), empero, reciben 0.143 (menos $124 \%$ ), lo que corresponde a la parte no explicada que es atribuible a la discriminación. En dicha ocupación, la única variable que tiene una relación positiva es la edad.

En lo que concierne a la educación, las mujeres, en promedio, tienen más años formales de educación. Además, es superior la proporción de mujeres que reside en el área urbana; más mujeres trabajan de manera formal y se autodenominan como no indígenas, en comparación con las proporciones correspondientes de los hombres.

La ocupación de técnicos y profesionales de nivel medio presenta un coeficiente de diferencia salarial logarítmica de 0.168 . Según la parte explicada, las mujeres deberían de ganar -0.030 (más $18 \%$ ) con sus atributos productivos, sin embargo, reciben 0.198 (menos $118 \%$ ), correspondiente a la parte no explicada atribuible a la discriminación. En dicha ocupación, las variables que tienen una ponderación positiva con la parte explicada es la edad y autoidentificarse como no indígena.

Las ocupaciones que se encuentran por debajo de dicho rango son en las que existe mayor igualdad: directores/as y operadores/as.

La ocupación de directores/as y gerentes muestra que existe una diferencia de 0.07 puntos logarítmicos y, según la parte explicada, las mujeres deberían de ganar -0.075 (108\% más) relacionado con sus cualidades personales, sin embargo, perciben 0.146 (208 \% menos), que corresponde a la parte no explicada que se atribuye a la discriminación. En el detalle de la composición de la parte explicada, se evidencia que la variable de edad, edad al cuadrado y el área de vivienda urbana tienen una relación positiva. Es importante mencionar que en dicha ocupación se desconoce la relación entre la diferencia salarial por contrato, esto, debido a la ausencia de observaciones de mujeres sin contrato.

En la ocupación de operadores/as de instalaciones y máquinas y ensambladores/as, las mujeres se encuentran mejor posicionadas, con una diferencia de -0.079 puntos logarítmicos en contra de los hombres; al analizar la parte explicada por las cualidades personales, se encontró que deberían de ganar-0.183 (más $231 \%$ ), y la parte no explicada fue de 0.103 (menos $131 \%$ ), también a favor de las mujeres. En la parte explicada, las variables que tienen una relación negativa en la diferencia 
salarial explicada es contar con contrato y autodenominarse como no indígena, y es mayor la proporción de las mujeres con contrato que los hombres, así como mujeres que se autodenominan como no indígenas. Mientras, se presenta una relación positiva entre la diferencia salarial explicada y residir en el área urbana.

Por otro lado, las ocupaciones que se encuentran por arriba del rango de la brecha salarial son aquellas que tienen una desigualdad mayor: las y los oficiales y trabajadores/as de servicios. La ocupación de oficiales operarios/as y artesanos/as de artes mecánicas y de otros oficios presenta una brecha salarial de 0.964 puntos logarítmicos, compuesta por una parte explicada que demuestra que las mujeres deberían de ganar 0.238 (más $25 \%$ ) y, sin embargo, reciben 0.726 (menos $75 \%$ ), lo que corresponde a la parte no explicada que es atribuible a la discriminación. En dicha ocupación, las variables de mayor ponderación de la parte explicada son la edad, etnicidad no indígena, contar con un contrato, el nivel educativo y, por último, residir en el área urbana.

Asimismo, la ocupación de trabajadores/as de los servicios y vendedores/as de comercios y mercados, con un coeficiente de diferencia de 0.567 puntos logarítmicos, demuestra que las mujeres deberían de percibir un 0.213 , es decir, $38 \%$ más, pero reciben 0.354 , lo que corresponde a $62 \%$ menos, correspondiente a la parte no explicada que se puede atribuir a la discriminación. Las variables con mayor peso en la parte explicativa son la edad y contar con un contrato; dicho sector tiene una proporción mayor de hombres que cuentan con un trabajo formal en comparación a las mujeres. 


\section{Conclusiones}

En Guatemala, existe una diferencia en el nivel salarial bastante marcada: el de los hombres es mayor con respecto al de las mujeres en todas las ocupaciones. La magnitud de la diferencia salarial varía por ocupación: el rango intercuartílico de razón salarial mujer-hombre es del $57 \%$ y $89 \%$, con media de $79 \%$. Se encuentra una mayor diferencia salarial entre hombres y mujeres en las ocupaciones de oficiales operarios/as y artesanos/as de artes mecánicas y de otros oficios, y en las y los trabajadores de los servicios de comercios y mercados. El caso contrario se observa en directores/as y gerentes, y operadores de instalaciones y máquinas y ensambladores/as, los cuales tienen la menor brecha.

Adicionalmente, al observar los salarios en Guatemala, en promedio, estos se encuentran por debajo de la capacidad adquisitiva de la Canasta Ampliada y cinco ocupaciones se encuentran por debajo de la Canasta Básica Alimentaria.

A partir de los modelos de los ingresos, se puede sugerir que en todas las ocupaciones, hombres y mujeres incrementan sus posibilidades de que su ingreso se eleve al aumentar la cantidad de años de educación formal. Asimismo, la investigación evidencia que la fuerza laboral femenina de la población ocupada se encuentra por debajo del $29 \%$ y su concentración es dentro de las ocupaciones con salarios bajos y medios. 


\section{Referencias}

Instituto Nacional de Estadística (INE). (Marzo de 2019). Canasta Básica Alimentaria (CBA) y Canasta Ampliada (CA) febrero de 2019. (Mayo de 2019). Encuesta Nacional de Empleo e Ingreso (ENEI) 1-2019. (Octubre de 2019). Cuántos somos. https://bit.ly/3uQ8opL

Ortiz, N. (2017). Discriminación salarial: brecha salarial entre hombres y mujeres del mercado laboral paraguayo. https://bit.ly/393IRCM 


\section{Bibliografía}

Becker, G. (1957). The Economics of Discrimination. The University of Chicago Press.

Bour, E. (2018). La Ecuación de J. Mincer.

Caamaño, E. (2001). La discriminación laboral indirecta. Revista de derecho (Valdivia), 1-15. https://bit.ly/2SoE1yc

Case, K. y Fair, R. (1997). Principio de Microeconomía. Pretince-Hall Hispanoamericana, S. A.

Castaño, C. (1999). Economía y género. Política y Sociedad, (31), 23-42.

Castrillón, J. D. (3 de octubre de 2019). Conceptos generales de economía. https://bit.ly/3cfsuln

Comisión Económica para América Latina y el Caribe (Cepal). (Julio de 1995). Descripción de los indicadores de ingresos y salarios y su implementación en el Uruguay.

Daczo, Z. (2012). Wage inequality and the gender wage gap: American Women swimming upstream? Universidad de Maryland.

Díaz, E. (2015). La desigualdad salarial entre hombres y mujeres. Alcances y limitaciones de la ley No. 20.348 para avanzar en justicia de género. Dirección de Trabajo.

Díaz, J. F. (2006). Incidencia del nivel de escolaridad y la experiencia laboral en el ingreso de los individuos de la población ocupada del área urbana para los sectores formal e informal de la economía durante el año 2003 [Tesis de grado, Universidad Rafael Landívar].

Ehrenberg, R. G. \& Smith, R. S. (2016). Modern labor economics: Theory and public policy. Routledge.

Espino, J. (2001). Dispersión salarial, capital humano y segmentación laboral en Lima. Documento de trabajo e investigación. Pontificia Universidad Católica del Perú.

Fernández, E. \& Agüera, J. (2012). La teoría de la segmentación del mercado de trabajo. Una reconsideración desde la perspectiva institucionalista y poskeynesiana [Tesis doctoral, Universidad de León, Departamento de Economía y Estadística]. 
Foro Económico Mundial. (2019). Comunicado de Más de una vida por delante: 100 años para alcanzar la paridad de género en el mundo. https://bit. ly/3w21xtr

Fuentes, J., Palma, A. \& Montero, R. (2005). Discriminación salarial por género en Chile: una mirada global. Estudios de Economía, 133-157. https://bit. ly/2S7AogA

González, P. (2001). La diferencia de ingresos entre hombres y mujeres: teoría, evidencia e implicaciones de política. Colección de estudios CIEPLAN, $34,101-152$.

Guil, A. (2008). Mujeres y ciencia: techos de cristal. Eccos Revista Científica, 213-231.

Gujarati, D. y Porter, D. (2009). Econometría (5. a edición). Editorial McGraw Hill.

Herrera, S. (2010). La importancia de la educación en el desarrollo: la teoría del capital humano y el perfil edad. Ingresos por nivel educativo en Viedma y Carmen de Patagones, Argentina. Repositorio Digital Institucional, Año VIII, 16(2), 9-26. https://bit.ly/3ciTods

Huard, A. (2003). La brecha salarial entre mujeres y hombres en Guatemala.

Instituto Nacional de Estadística (INE). (Junio de 2018). Encuesta Nacional de Empleo e Ingreso (ENEI) 1-2018.

Kapsos, S. (2008). The gender wage gap in Bangladesh. Publications of the International Labour Office.

López, C. P. (2006). Problemas resueltos de econometría. Editorial Paraninfo.

Ministerio de Trabajo. (2008). Código de trabajo de Guatemala.

Molina, M. (16 de septiembre de 2019). La discusión que no hemos tenido: empleo y desigualdad de género. https://bit.ly/3cgn2Pg

Moner, R. (2008). «Segmentación de los mercados de trabajo y relaciones laborales. El sindicalismo ante la acción colectiva». En Cuadernos de Relaciones Laborales (pp. 123-148).

ONU Mujeres. (16 de septiembre de 2019). Las mujeres en el cambiante mundo del trabajo. https://bit.ly/3cfon5V

Organización Internacional del Trabajo (OIT). (2010a). Informe mundial sobre salarios 2010/2011. Políticas salariales en tiempos de crisis. (2010b). Resolución sobre la actualización de la Clasificación Internacional Uniforme de Ocupaciones. https://bit.ly/3cgnhtx 
(2016a). InfoStories: La igualdad de remuneración como solución a la discriminación basada en el sexo. https://bit.ly/3wY8taG

(2016b). Las mujeres en el trabajo: Tendencias de 2016.

Organización para la Cooperación y el Desarrollo Económicos (OCDE). (2018). La búsqueda de la igualdad de género. Una batalla cuesta arriba. (16 de septiembre de 2019). Gender wage gap. https://bit.ly/3ceuomc

Parkin, M. (2014). Economía. Pearson.

Pernaut, M. (2008). Introducción a la Teoría Económica. UCAR.

Posso, C. (2010). Calidad del empleo y segmentación laboral: un análisis para el mercado laboral colombiano. Revista Desarrollo y Sociedad, 191-234.

Sefarini, V. (2008). La economía y el género: una relación compleja. Clacso. https://bit.ly/zci1Sza

Sepúlveda, B., Algarra, Á. \& Ramos, R. (2012). Explicaciones Teóricas de la Discriminación de la Mujer en el Mercado de Trabajo: Fundamentos Microeconómicos. https://bit.ly/3pmkEM1

Sistemas de las Naciones Unidas en Guatemala. (8 de marzo de 2017). Las Mujeres Guatemaltecas, hacia un planeta 50-50. https://bit.ly/3iiyO7w

Smith, A. (1996). La Riqueza de las Naciones (C. R. Braun, trad.). Alianza Editorial Madrid.

Spencer, M. H. (1993). Economía contemporánea. Reverté, S. A.

Thurow, L. (1972). Education and Income Inequality. Readings in Labour Economics. 